Pacific Journal of Mathematics

COMMUTATIVE RINGS WHOSE HOMOMORPHIC IMAGES 


\title{
COMMUTATIVE RINGS WHOSE HOMOMORPHIC IMAGES ARE SELF-INJECTIVE
}

\author{
LAWRENCE S. LEVY
}

\begin{abstract}
Dedekind domains are characterized among integral domains by the property that every ideal be a projective module. The most naive dual characterization-that every homomorphic image of $R$ be an injective module-is false. In fact, a domain with this property would have to be a field. An injectivity property that works, in the noetherian case, is the property that every proper homomorphic image be a self-injective ring. The main result of this note is:
\end{abstract}

THEOREM. Let $R$ be a commutative, noetherian ring (with identity). Every proper homomorphic image of $R$ is a self-injective ring if and only if

(1) $R$ is a Dedekind domain, or

(2) $R$ is a principal ideal ring with descending chain condition, or

(3) $R$ is a local ring whose maximal ideal $M$ has composition length 2 and satisfies $M^{2}=0$.

A more complete description of the rings of type (3) is given in Remark (ii) below.

Lemma. Suppose that $M^{2}=0$ for some maximal ideal $M$ of $a$ self-injective, commutative ring $R$. Then $0 \leqq M \leqq R$ are all of the ideals of $R$.

Proof. For $x$ in $R$ let ann $x$ (the annihilator of $x$ ) be the ideal of all $b$ in $R$ such that $b x=0$. If $x$ is a nonzero element of $M$, then $M \cong$ ann $x$ since $M^{2}=0$; and since $R$ has an identity, $R \neq$ ann $x$. Maximality of $M$ therefore shows that $M=$ ann $x$ so that $R x \cong$ $R /$ ann $x=R / M$ (as modules). Hence, for any two nonzero elements $x, y$ of $M$ we have $R x \cong R y$.

Injectivity of the $R$-module $R$ implies that any isomorphism of $R x$ onto $R y$ can be extended to a homomorphism of $R$ into itself. Suppose that, in such an extended map, $1 \rightarrow u$. Then $R x u=R y$ so that $R x \supseteqq$ $R y$ for every nonzero $x$ and $y$ in $M$. This shows that $M$ has no proper submodules.

Now let $N$ be any ideal $\neq 0$ or $M$. Then since there are no ideals between 0 and $M$, and since $M$ is maximal, we have $R=M+N$. Multiplying by $M$ and recalling that $M^{2}=0$ we get $M=M N \cong N$ so that $N=R$. 
Proof of the theorem. Suppose that all of the proper homomorphic images of $R$ are self-injective rings, and consider first the case that $R$ is a domain. If $M$ is a maximal ideal of $R$, the ring $R / M^{2}$ must be self-injective and the image $\bar{M}$ of $M$ in $R / M^{2}$ satisfies $\bar{M}^{2}=0$; so by our lemma, $R$ has no ideals between $M$ and $M^{2}$. But a theorem of I. S. Cohen [2, Theorem 8] states that a noetherian domain with this last property for every maximal ideal $M$ must be a Dedekind domain. Thus we have obtained rings of type(1).

Suppose next that $R$ is not a domain. Then 0 is not a prime ideal. Observe that every prime ideal $P$ must be maximal: The integral domain $R / P$ is, by hypothesis, an injective, hence divisible $R / P$-module [1, Chap. 7, Prop. 12]; that is, a field. But a noetherian commutative ring in which every prime ideal is maximal must satisfy the DCC (descending chain condition) [2, Theorem 1], and hence be the direct sum $R=R_{1} \oplus \cdots \oplus R_{n}$ of local rings, with DCC [6, Chap. 4, Theorem 3.3].

Let $M_{i}$ be the maximal ideal of $R_{i}$. Again we consider two cases. Suppose first that either $n>1$, or $n=1$ but $M_{1}^{2} \neq 0$. Then for each $i, R /\left(M_{i}^{2}+\sum_{j \neq i} R_{j}\right) \cong R_{i} / M_{i}^{2}$ is a self-injective ring. The lemma (applied to $R_{i} / M_{i}^{2}$ ) tells us that for any $m_{i}$ in $M_{i}$ but not in $M_{i}^{2}$ we have $M_{i}=R_{i} m_{i}+M_{i}^{2}=R_{i} m_{i}+\left(\operatorname{rad} R_{i}\right) M_{i}$; and Nakayama's lemma then shows that $M_{i}=R_{i} m_{i}$. Thus $R_{i}$ is a noetherian commutative ring in which every maximal ideal is principal, and a theorem of Kaplansky asserts that any such ring must be a PIR (principal ideal ring) [3, Theorem 12,3]. Thus each $R_{i}$ and hence $R$ itself is of type (2).

Finally, suppose that $R$ is a local ring with DCC whose maximal ideal $M$ satisfies $M^{2}=0$. We can suppose that $M$ has composition length at least 2 , since otherwise $R$ would be a PIR. By the DCC, $M$ contains a minimal ideal $N$ of $R$. By the lemma, the nonzero ideal $M / N$ of the self-injective ring $R / N$ has composition length 1 . Hence $M$ has composition length 2 . Thus $R$ is of type (3) and the proof of half of the theorem is done.

Conversely, suppose that $R$ is of type (1), (2), or (3). Observe that the proper homomorphic images of all three types of rings are all PIR's with DCC, and that every ring of this last type is the direct sum of rings $R$ which have exactly one composition series [3, Theorem 13,3]:

$$
R \supset R m \supset R m^{2} \supset R m^{3} \supset \cdots \supset R m^{t}=0
$$

It is therefore sufficient to show that the ring in (4) is self-injective. To do this let $f$ be a homomorphism of $R m^{s}$ into $R$. Then the composition length of $f\left(R m^{s}\right)$ cannot exceed that of $R m^{s}$, so that the 
fact that (4) contains all the ideals of $R$ shows that $f\left(R m^{s}\right) \subseteq R m^{s}$. Consequently, $f\left(m^{s}\right)=m^{s} x$ for some $x$ in $R$. The map $r \rightarrow r x(r$ in $R)$ is then an extension of $f$ to a homomorphism of $R$ into itself, showing that $R$ is an injective $R$-module and completing the proof of the theorem.

The following consequence (actually, restatement) of the theorem is the converse of an old lemma which is the starting point for some accounts of the theory of finite abelian groups. We define the order ideal of an element $m$ of an $R$-module $M$ to be $\{r \in R: r m=0\}$, and we say that $M$ has bounded order if $r M=0$ for some $r \neq 0$.

COROLlaRY. Let $R$ be a commutative noetherian ring and suppose whenever $m$ is an element with minimal order ideal in an $R$-module $M$ of bounded order, that $R m$ is a direct summand of $M$. Then $R$ is of type (1), (2), or (3).

For a proof, let the ring $\bar{R}$ be a proper homomorphic image of $R$, and let $M$ be any $\bar{R}$-module which contains $\bar{R}$. Then $M$ has bounded order as an $R$-module, and the identity of $\bar{R}$ is an element with minimal order ideal in the $R$-module $M$. Hence, by hypothesis, $\bar{R}$ is a direct summand of $M$. We have thus shown that $\bar{R}$ is a direct summand of every $\bar{R}$-module which contains it, and hence is selfinjective. The theorem now completes the proof of the corollary.

REMARKS. (i) The hypothesis that $R$ be noetherian cannot be dropped from the theorem. For an example, let $F$ be a field and $x$ an indeterminate; and let $W$ be the family of all well-ordered sets $\{i\}$ of nonnegative real numbers, the order relation being the natural order of the real numbers. Then let $R$ be the set of all "formal power series"

$$
\sum_{i \in\{i\}} a_{i} x^{i}
$$

with $a_{i}$ in $F$ and $\{i\}$ in $W$. Then $R$ is a nonnoetherian domain whose finitely generated ideals are principal and whose proper homomorphic images are self-injective.

We will need the facts that $R$ is actually a ring and that every element of $R$ whose constant term is nonzero is invertible in $R[4$, part I]. It follows that every nonzero element of $R$ has the form $x^{b} u$ where $u$ is invertible in $R$. This implies that $R$ has only two types of nonzero ideals: The principal ideals $\left(x^{b}\right)$, and those of the form $\left(x^{>b}\right)=\left\{x^{c} u: c>b\right.$ and $u$ is invertible or zero $\}$. 
Let $S=R / J$ where $J \neq 0$ and let $y=x+J$ so that $S$ can be considered at the collection of "formal power series"

$$
\sum_{i \in\{i\}} a_{i} y^{i}
$$

with $a_{i}$ in $F,\{i\}$ in $W$, and (I) $y^{b}=0$ if $J=\left(x^{b}\right)$, or (II) $y^{c}=0$ for $c>b$ if $J=\left(x^{>b}\right)$. Observe that for $c \leqq b$

$$
\text { (if } J=\left(x^{b}\right) \text { ) }
$$$$
\operatorname{ann}\left(y^{c}\right)=\left(y^{b-c}\right)
$$$$
\operatorname{ann}\left(y^{>c}\right)=\left(y^{b-c}\right)
$$$$
\text { (if } J=\left(x^{>b}\right) \text { ) }
$$$$
\operatorname{ann}\left(y^{c}\right)=\left(y^{>b-c}\right)
$$$$
\operatorname{ann}\left(y^{>c}\right)=\left(y^{b-c}\right) \text {. }
$$

From (5) and (6) it follows that whether $S$ is of type (I) or (II), the principal ideals of $S$ satisfy ann ann $\left(y^{c}\right)=\left(y^{c}\right)$.

To see that $S$ is self-injective, let $f$ be an $S$-homomorphism of an ideal $K$ of $S$ into $S$. If $K=\left(y^{c}\right)$, then $($ ann $K) f(K)=f(0)=0$ so that $f(K) \subseteq$ ann ann $K=K$ so that $f\left(y^{c}\right)=y^{c} p$ for some $p$ in $S$. Thus $f$ can be extended to the endomorphism $s \rightarrow s p$ of $S$.

Next, let $K=\left(y^{>c}\right)$, and choose an infinite sequence $c(1)>c(2)>$ $c(3)>\cdots$ such that $\lim _{i \rightarrow \infty} c(i)=c$. Then $K=\cup_{i=1}^{\infty}\left(y^{c(i)}\right)$. For each $i$ the previous paragraph shows that we can choose a "power series" $p_{i}$ such that $f\left(y^{c(i)}\right)=y^{c(i)} p_{i}$. If $j>i$ so that $y^{c(i)}=y^{c(i)-c(j)} y^{c(j)}$ the fact that $f$ is an $S$-homomorphism shows that $f\left(y^{c(i)}\right)=y^{c(i)} p_{j}$ so that (since also $f\left(y^{c(i)}\right)=y^{c(i)} p_{i}$ ) we have $p_{j}-p_{i} \in$ ann $\left(y^{c(i)}\right)$. If (5) holds, this means that all the terms of $p_{i}$ of degree $<b-c(i)$ are equal to the terms of the same degree of $p_{j}$ while the terms of higher degree do not affect the products $y^{c(i)} p_{i}$ and $y^{c(i)} p_{j}$. A similar statement is true if (6) holds. Thus we can assemble a single "power series" $p$ such that $\left(p-p_{i}\right) y^{c(i)}=0$ for all $i$ (It must be verified that the collection of exponents appearing in $p$ is well-ordered so that $p$ is in $S$ ). Then the map $s \rightarrow s p$ extends $f$ to an endomorphism of $S$. We have now shown $S$ to be self-injective.

(ii) The rings of type (3) in the theorem can be characterized as the following type of combination of PIR's: Let $\bar{R}$ be a field, and let $R_{i}$ be a local PIR with maximal ideal $M_{i} \neq 0$ such that $R_{i} / M_{i} \cong \bar{R}$ and $M_{i}^{2}=0(i=1,2)$. Note that $M_{i}$ is the only proper ideal of $R_{i}$. Choose pair of homomorphisms $k_{i}: R_{i}$ onto $\bar{R}$ and let $R=\left\{\left(r_{1}, r_{2}\right): k_{1}\left(r_{1}\right)=\right.$ $\left.k_{2}\left(r_{2}\right)\right\}$.

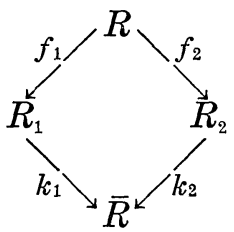


To see that $R$ is of type (3), note that an element $\left(r_{1}, r_{2}\right)$ of $R$ is a nonunit if and only if $k_{1}\left(r_{1}\right)=0\left(=k_{2}\left(r_{2}\right)\right)$. Hence $R$ is a local ring. Its maximal ideal is readily seen to be $M=M_{1} \oplus M_{2}$ and hence has composition length 2 and satisfies $M^{2}=0$.

Conversely, let $R$ be of type (3). Then since $M^{2}=0, M$ is a vector space over the field $\bar{R}=R / M$, and this space has dimension 2 . Hence $M$ is the direct sum of two minimal ideals $M_{1}$ and $M_{2}$ of $R$. The map $f: r \rightarrow\left(r+M_{1}, r+M_{2}\right)$ of $R$ into $R_{1}\left(=R / M_{1}\right) \oplus R_{2}\left(=R / M_{2}\right)$ is a monomorphism (see the diagram). Finally, define $k_{i}: R_{i}$ onto $\bar{R}$ by $k_{i}\left(r+M_{i}\right)=r+M$. Then it is straightforward to verify that $R \cong f(R)=\left\{\left(r+M_{1}, s+M_{2}\right): r+M=s+M\right\}=\left\{\left(r_{1}, r_{2}\right): k_{1}\left(r_{1}\right)=k_{2}\left(r_{2}\right)\right\}$.

(iii) Finally, it seems fitting to mention a related theorem by Barbara Osofsky [5] which states that the only rings $R$ (commutative or not) having the property that all of their (left $R$-) homomorphic images are injective $R$-modules are the semi-simple rings with DCC.

\section{REFERENCES}

1. H. Cartan and S. Eilenberg, Homological algebra, Princeton Univ. Press, Princeton, N. J. (1956)

2. I. S. Cohen, Commutative rings with restricted minimum condition, Duke J. Math. 17 (1950), p. 27-42.

3. I. Kaplansky, Elementary divisors and modules, Trans Amer. Math. Soc. 66(1949).

4. B. H. Neuman, On ordered division rings, Trans. Amer. Math. Soc. 66 (1949), p. 202-252.

5. B. L. Osofsky, Rings all of whose finitely generated modules are injective, Pacific J. Math. 14 (1964), p. 645-650.

6. O. Zariski and P. Samuel, Commutative algebra, Vol. 1, Van Nostrand, Princeton, N. J., (1958).

Received February 26, 1965. This research was partially supported by the National Science Foundation under Grant GP-3431. The author is greatly indebted to the referee who found, and corrected, an error in the original version of this paper.

UNIVERSITY OF WISCONSIN

UNIVERSITY OF CHICAGo 



\section{PACIFIC JOURNAL OF MATHEMATICS}

H. SAMELSON

Stanford University Stanford, California

R. M. BLUMENTHAL

University of Washington

Seattle, Washington 98105

\section{EDITORS}

\author{
*J. DugundJI \\ University of Southern California \\ Los Angeles, California 90007 \\ RICHARD ARENS \\ University of California \\ Los Angeles, California 90024
}

\section{ASSOCIATE EDITORS}

E. F. BeCKENBACH

B. H. NEUMANN

F. WOLF

K. YosidA

\section{SUPPORTING INSTITUTIONS}

UNIVERSITY OF BRITISH COLUMBIA

CALIFORNIA INSTITUTE OF TECHNOLOGY

UNIVERSITY OF CALIFORNIA

MONTANA STATE UNIVERSITY

UNIVERSITY OF NEVADA

NEW MEXICO STATE UNIVERSITY

OREGON STATE UNIVERSITY

UNIVERSITY OF OREGON

OSAKA UNIVERSITY

UNIVERSITY OF SOUTHERN CALIFORNIA
STANFORD UNIVERSITY

UNIVERSITY OF TOKYO

UNIVERSITY OF UTAH

WASHINGTON STATE UNIVERSITY

UNIVERSITY OF WASHINGTON

AMERICAN MATHEMATICAL SOCIETY CHEVRON RESEARCH CORPORATION TRW SYSTEMS

NAVAL ORDNANCE TEST STATION 


\section{Pacific Journal of Mathematics}

Vol. 18, No. 1

March, 1966

Edward Joseph Barbeau, Semi-algebras that are lower semi-lattices ...... 1

Steven Fredrick Bauman, The Klein group as an automorphism group

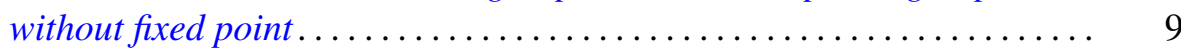

Homer Franklin Bechtell, Jr., Frattini subgroups and $\Phi$-central groups .... 15

Edward Kenneth Blum, A convergent gradient procedure in prehilbert

spaces ............................................

Edward Martin Bolger, The sum of two independent exponential-type random variables ...................................

David Wilson Bressler and A. P. Morse, Images of measurable sets .......

Dennison Robert Brown and J. G. LaTorre, A characterization of uniquely

divisible commutative semigroups........................

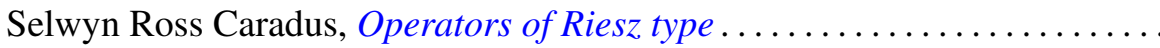

Jeffrey Davis and Isidore Isaac Hirschman, Jr., Toeplitz forms and ultraspherical polynomials ............................

Lorraine L. Foster, On the characteristic roots of the product of certain rational integral matrices of order two ......................

Alfred Gray and S. M. Shah, Asymptotic values of a holomorphic function



Sidney (Denny) L. Gulick, Commutativity and ideals in the biduals of



G. J. Kurowski, Further results in the theory of monodiffric functions

Lawrence S. Levy, Commutative rings whose homomorphic images are self-injective .

Calvin T. Long, On real numbers having normality of order $k$....

Bertram Mond, An inequality for operators in a Hilbert space. ...

John William Neuberger, The lack of self-adjointness in three-point boundary value problems ........................

C. A. Persinger, Subsets of $n$-books in $E^{3}$

Oscar S. Rothaus and John Griggs Thompson, A combinatorial problem in the symmetric group ............................... 175

Rodolfo DeSapio, Unknotting spheres via Smale .................. 179

James E. Shockley, On the functional equation

$$
F(m n) F((m, n))=F(m) F(n) f((m, n)) \ldots \ldots \ldots
$$

Kenneth Edward Whipple, Cauchy sequences in Moore spaces ... 